\title{
Evaluation of byproducts from the wine-making industry by identification of bioactive compounds
}

\author{
C.E. Luchian ${ }^{1}$, E.C. Scutaraşu ${ }^{1}$, L.C. Colibaba ${ }^{1}$, V. V. Cotea ${ }^{1}$, L. Vlase ${ }^{2}$, and A.M. Toiu ${ }^{2}$ \\ ${ }^{1}$ University of Agricultural Sciences and Veterinary Medicine "Ion Ionescu de la Brad", Iaşi, Romania \\ 2 "Iuliu Haţieganu" University of Medicine and Pharmacy, V. Babeş Street, Cluj Napoca, Romania
}

\begin{abstract}
Byproducts obtained after wine production constitute an important source for the extraction of antioxidant compounds. Phenolic compounds showed a wide spectrum of biological actions for the human health, often associated with neuroprotective, cardioprotective, antimicrobial and other health-related effects. The aim of the present study was to determine the phenolic compounds content and antioxidant activity of grape marc from selected grapes from Iaşi vineyard. The total phenolic content of the extracts was measured using the Folin-Ciocâlteu method, with some modifications. The DPPH (2,2-diphenyl-1-picrylhydrazyl) assay was used to evaluate radical scavenging activity, by bleaching of purple methanolic solution of the stable radical. All extracts were able to reduce DPPH radical with different degrees of scavenging activity. The highest radical scavenging activity was determined for the extract obtained from Merlot and Fetească Neagră $(98.142 \mu \mathrm{g} / \mathrm{mL}$, and $115.78 \mu \mathrm{g} / \mathrm{mL}$ respectively), with positive correlation between scavenging activity on DPPH and total phenolic content. The total phenolic content varied among the analysed extracts, with a higher concentration in extracts obtained from Merlot, Fetească Neagră and Traminer rosé $(2.42 \mathrm{GAE} / \mathrm{mL}$, 1.45 GAE/mL and 1.24 GAE/mL respectively), followed by Sauvignon Blanc and Muscat Ottonel extracts with comparable amounts. The FRAP (ferric reducing antioxidant power) method relies on the color change of a complex with $\mathrm{Fe}^{+3}$ ion of the 2,4,6-tri(2-pyridyl) - 1,3,5-triazine (TPTZ) radical due to the reduction of the ferric ion to the ferrous iron $\left(\mathrm{Fe}^{+2}\right)$ in this complex. The highest radical scavenging activity was determined in the extract obtained from Merlot and Fetească Neagră $(1164.22 \mu \mathrm{M}$ Trolox/100 mL and $782.09 \mu \mathrm{M}$ Trolox/100 $\mathrm{mL}$ respectively), a positive correlation between scavenging activity determined by FRAP method and total phenolic content being observed.Statistical analysis showed a close relationship between content of phenolic compounds and antioxidant capacity, the marc of Merlot and Fetească Neagră had the highest contents of anthocyanins. The results suggested that grape pomace may be exploited as antioxindant source.
\end{abstract}

\section{Introduction}

In the last few years, reducing the environmental action of industrial wastes has been of increasing concern. Grapes represent one of the world's largest fruits crops, and even winemaking byproducts, such as marc and stalks, are rich in phenolic compounds [1]. Byproducts obtained after wine production constitute an important source for the extraction of antioxidant compounds. Phenolic compounds showed a wide spectrum of biological actions for the human health, often associated with neuroprotective, cardioprotective, antimicrobial and other health-related effects. These compounds can be used as anti-inflammatory, anti-cancer and anti-aging agents. The phenolic compounds found in wine can act as antioxidants, while the wastes resulted in wine production are also characterized by high contents of phenolic compounds due to their incomplete extraction during wine production. The seeds and the pomace represent a cheap source of antioxidant compounds. Dried seed extract contains the higher level of antioxidant compounds while dried pulp contains the lowest [2].

The consumption of safe food and beverages, with high levels of phenolics antioxidants compounds, can reduce the risks of some chronic diseases and oxidative stress. Their use in nutraceutical products and healthy food or drinks has determined the need for obtaining antioxidants from wine byproducts. Millions of tons of wastes are generated every year by the winemaking production [3], and very small quantities of these are reused, the majority being generally dumped or used for animal feed and compost, without any pre-treatment. Part of the grape pomace is used for distillation but minimum amounts of components are recovered [4]. The aim of the present study was to determine the phenolic compounds content and antioxidant activity of grape marc from selected grapes varieties from Iaşi vineyard, a northern Romanian area.

\section{Materials and methods}

The necessary plant material for the study was represented by the grape varieties Sauvignon Blanc, Traminer, Busuioacă de Bohotin, Cabernet Sauvignon, Merlot, Fetească Neagră and Muscat Ottonel harvested in autumn 2017 at full maturity from Iaşi vineyard - Bucium region and Bohotin region. After the quantitative and qualitative reception, the grapes were subjected to crushing and 
Table 1. Extracts obtained from grape varieties.

\begin{tabular}{cc}
\hline EXTRACT NUMBER & VARIETY \\
\hline 1 & Merlot \\
2 & Cabernet Sauvignon \\
3 & Busuioaca de Bohotin \\
4 & Muscat Ottonel \\
5 & Fetească Neagră \\
6 & Traminer \\
7 & Sauvignon Blanc \\
\hline
\end{tabular}

desteming followed by maceration ( 24 hours for whites and 5 days for reds at $10^{\circ} \mathrm{C}$ ) and then pressed with a pneumatic press. The grape pomace resulted from pressing was dried and frozen in plastic packaging.

\subsection{Determination of total polyphenolic content}

The total phenolic content (TPC) of the extracts was measured using the Folin-Ciocalteu method, with some modifications. The absorbance was measured at $760 \mathrm{~nm}$, using a JASCO UV-VIS spectrophotometer. Standard curve was prepared by using different concentrations of gallic acid. TPC was expressed as $\mathrm{mg}$ gallic acid/ $\mathrm{mL}$ extract (mg GAE/mL extract).

\subsection{DPPH assay}

The DPPH (2,2-diphenyl-1-picrylhydrazyl) assay was used to evaluate radical scavenging activity, by bleaching of purple methanolic solution of the stable radical. The measurement of the antioxidant effect is calculated according to the DPPH absorbtion by antioxidants. $20 \mu \mathrm{l}$ of diluted extracts were added to $980 \mu \mathrm{LPPH}$ solution $(100 \mu \mathrm{M})$. The decrease in absorbance was measured at $517 \mathrm{~nm}$, using a UV-VIS JASCO V-530 spectrophotometer after $30 \mathrm{~min}$ incubation period. Both hydrophilic and lipophilic synthetic antioxidants, quercetin and butylated hydroxytoluene (BHT) were used as standards. The percentage inhibition of DPPH radical after adding individual samples was calculated using the following equation: $\mathrm{I}=100\left(\mathrm{~A}_{\mathrm{c}}-\mathrm{A}_{\mathrm{s}}\right) / \mathrm{A}_{\mathrm{c}}$, where $\mathrm{I}-\mathrm{DPPH}$ inhibition (\%), $\mathrm{A}_{\mathrm{c}}-$ absorbance of control sample, $\mathrm{A}_{\mathrm{s}}-$ absorbance of tested sample. Antioxidant activity was also expressed as inhibitory concentration $\mathrm{IC}_{50}$, defined as the concentration of the sample required to cause a $50 \%$ decrease in initial DPPH radical absorbance. $\mathrm{IC}_{5} 0$ values in DPPH assay were calculated graphically. All experiments were performed in triplicate [5-7].

\subsection{Ferric reducing antioxidant power assay}

FRAP assay is a novel method for assessing "antioxidant power". The FRAP (ferric reducing antioxidant power) method relies on the change in the color of a complex with $\mathrm{Fe}^{+3}$ ion of the 2,4,6-tri(2-pyridyl)-1,3,5-triazine (TPTZ) radical due to the reduction of the ferric ion to the ferrous iron $\left(\mathrm{Fe}^{+2}\right)$ in this complex. Trolox was used as reference. A curve absorbance was built according to Trolox mass, the correlation coefficient $\left(\mathrm{R}^{2}\right)$ for this curve being 0.992 . The final results were converted to $\mu \mathrm{M}$ Trolox equivalents $/ 100 \mathrm{~mL}$ extract $[8,9]$.
Table 2. TPC in analysed extracts ( \pm SD).

\begin{tabular}{cc}
\hline Sample & TPC $(\mathbf{m g ~ G A E} / \mathbf{m L})$ \\
\hline 1 & $2.421 \pm 0.09$ \\
2 & $0.642 \pm 0.03$ \\
3 & $0.404 \pm 0.02$ \\
4 & $0.771 \pm 0.05$ \\
5 & $1.452 \pm 0.07$ \\
6 & $1.242 \pm 0.05$ \\
7 & $0.884 \pm 0.06$ \\
\hline
\end{tabular}

Table 3. Antioxidant activity of extracts using DPPH method.

\begin{tabular}{cc}
\hline Sample & $\mathbf{I C}_{\mathbf{5 0}}(\boldsymbol{\mu g} / \mathbf{m L})$ \\
\hline 1 & $98.142 \pm 4.31$ \\
2 & $134.48 \pm 5.27$ \\
3 & $144.9 \pm 6.41$ \\
4 & $132.17 \pm 5.11$ \\
5 & $115.78 \pm 4.29$ \\
6 & $119.86 \pm 4.35$ \\
7 & $127.96 \pm 4.74$ \\
Quercetin & $5.59 \pm 0.13$ \\
BHT & $15.88 \pm 1.06$ \\
\hline
\end{tabular}

Note: Values are expressed as the mean $\pm \operatorname{SD}(n=3)$.

\section{Results and discussion}

\subsection{Polyphenols analysis}

The total phenolic content varied considering the analysed extracts, with higher concentration in extract 1 (Merlot), 5 (Fetească Neagră) and 6 (Traminer) $(2.42 \mathrm{GAE} / \mathrm{mL}$, $1.45 \mathrm{GAE} / \mathrm{mL}$, and $1.24 \mathrm{GAE} / \mathrm{mL}$ respectively), followed by 7 (Sauvignon Blanc) and 4 (Muscat Ottonel) extracts with comparable amounts. obtained concentrations of total polyphenols are presented in Table 2.

\subsection{Antioxidant activity assay by DPPH method}

In order to evaluate the ability of extracts and synthetic antioxidants quercetin and BHT to donate a hydrogen atom, the stable free radical DPPH was used. All extracts obtained from natural products were able to reduce DPPH radical with different degrees of scavenging activity. A lower $\mathrm{IC}_{50}$ value represents a higher effect, thus a better antioxidant activity.

The results obtained for the evaluation of the antioxidant activity using the DPPH assay are presented in Table 3.

Merlot extract (sample 1) had the strongest antioxidant activity with $\mathrm{IC}_{50}$ value of $98.142 \mu \mathrm{g} / \mathrm{mL}$. All analysed extracts showed lower DPPH scavenging activity than both quercetin and BHT. The highest radical scavenging activity was determined for extracts 1 (Merlot) and 5 (Fetească Neagră) $(98.142 \mu \mathrm{g} / \mathrm{mL}$, and $115.78 \mu \mathrm{g} / \mathrm{mL}$ respectively), with positive correlation between scavenging activity on DPPH and total phenolic content. The results are related to the presence of higher amounts of phenolic compounds and indicate that different active principles can contribute to antioxidant properties of natural products. The various antioxidant properties these extracts may be due to the variability of composition and content in phytochemicals, and the method used for extraction.

Considering the obtained results, the following order in antioxidant activity could be established: $3<2<4<$ $7<6<5<1<$ BHT $<$ quercetin. According to this 
Table 4. Antioxidant activity of extracts using FRAP method.

\begin{tabular}{cc}
\hline Sample & $\boldsymbol{\mu}$ M Trolox $/ \mathbf{1 0 0} \mathbf{~ m L}$ \\
\hline 1 & $1164.22 \pm 12.46$ \\
2 & $359.75 \pm 6.84$ \\
3 & $186.09 \pm 5.58$ \\
4 & $406.17 \pm 7.53$ \\
5 & $782.09 \pm 9.31$ \\
6 & $473.73 \pm 8.27$ \\
7 & $434.35 \pm 8.01$ \\
Trolox & $2073.91 \pm 26.08$ \\
\hline Note: Values are the mean $\pm \operatorname{SD~}(n=3)$.
\end{tabular}

method, the analysed extracts showed weak antioxidant effects [6].

\section{Ferric reducing antioxidant power (FRAP) assay}

The results obtained for the evaluation of the antioxidant activity using the FRAP assay are presented in Table 4.

The highest scavenging activity was determined for extract 1-Merlot and 5-Fetească Neagră $(1164.22 \mu \mathrm{M}$ Trolox $/ 100 \mathrm{~mL}$, and $782.09 \mu \mathrm{M}$ Trolox $/ 100 \mathrm{~mL}$ respectively), and a positive correlation between scavenging activity determined by FRAP method and total phenolic content was observed. Using the FRAP assay, the following order in antioxidant activity was found: $3<2<$ $4<7<6<5<1<$ Trolox, which could be correlated with the results obtained by DPPH assay.

\section{Conclusions}

Statistical analysis showed a close relationship between content of phenolic compounds and antioxidant capacity, the marc of Merlot and Fetească Neagră had the highest contents of anthocyanins. The results suggested that grape pomace may be exploited as antioxindant source.

All extracts were able to reduce DPPH radical with different degrees of scavenging activity. The highest radical scavenging activity was determined for the extract obtained from Merlot and Fetească Neagră, with positive correlation between scavenging activity on DPPH and total phenolic content.

The total phenolic content varied among the analysed extracts, with a higher concentration in extracts obtained from Merlot, Fetească Neagră and Traminer rosé, followed by Sauvignon Blanc and Muscat Ottonel extracts with comparable amounts.

The highest radical scavenging activity was determined in the extracts obtained from Merlot and Fetească Neagră, a positive correlation between scavenging activity determined by FRAP method and total phenolic content being observed.

The results obtained showed that the varieties studied have good potential as source of antioxidant compounds that could be applied as natural antioxidants in food and cosmetic products, in order the increase their shelf life.

The research was funded by the PN-III-P1-1.1-TE-2016-2038 project, nr. 77/8.05.2018.

\section{References}

[1] G. Spigno, D.M. De Faveri, J. Food Eng. 78, 793 (2005)

[2] J.A. Perez-Serradilla, M.D. Luque de Castro, Food Chem. 124, 1652 (2010)

[3] G. Spigno, L. Tramelli, D.M. De Faveri, J. Food Eng. 81, 200 (2006)

[4] N. Rangkadilok, S. Sitthimonchai, L. Worasuttayangkurn, C. Mahidol, M. Ruchirawat, J. Satayavivad, Food Chem. Toxicol. 45, 328 (2006)

[5] G. Ruberto, A. Renda, C. Daquino, V. Amico, C. Tringali, N. de Tommasi, Food Chem. 100, 203 (2007)

[6] A. Toiu, A. Mocan, L. Vlase, A.E. Pârvu, D.C. Vodnar, A.M. Gheldiu, C. Moldovan, I. Oniga, Front Pharmacol. 9, 1 (2018)

[7] J.H. Andriamadio, L.H. Rasoanaivo, D. Benedec, L. Vlase, A.M. Gheldiu, M. Duma, Nat. Prod. Res. 29, 2188 (2015)

[8] C.E. Pop, A.E. Pârvu, D.C. Vodnar, M. Tarcea, A.M. Toiu, L. Vlase, Farmacia 54, 624 (2017)

[9] K. Thaipong, U. Boonprakob, K. Crosby, L. Cisneros-Zevallos, D. Hawkins Byrne, J. Food Compost. Anal. 19, 669 (2006) 